\title{
Canonical Wnt signaling induces BMP-4 to specify slow myofibrogenesis of fetal myoblasts
}

Kazuki Kuroda ${ }^{1,4}$, Shihuan Kuang ${ }^{1,2}$, Makoto M Taketo ${ }^{3}$ and Michael A Rudnicki ${ }^{1 *}$

\begin{abstract}
Background: The Wnts are secreted proteins that play important roles in skeletal myogenesis, muscle fiber type diversification, neuromuscular junction formation and muscle stem cell function. How Wht proteins orchestrate such diverse activities remains poorly understood. Canonical Wnt signaling stabilizes $\beta$-catenin, which subsequently translocate to the nucleus to activate the transcription of TCF/LEF family genes.

Methods: We employed TCF-reporter mice and performed analysis of embryos and of muscle groups. We further isolated fetal myoblasts and performed cell and molecular analyses.

Results: We found that canonical Wnt signaling is strongly activated during fetal myogenesis and weakly activated in adult muscles limited to the slow myofibers. Muscle-specific transgenic expression of a stabilized

$\beta$-catenin protein led to increased oxidative myofibers and reduced muscle mass, suggesting that canonical Wnt signaling promotes slow fiber types and inhibits myogenesis. By TCF-luciferase reporter assay, we identified Wnt-1 and Wnt-3a as potent activators of canonical Wnt signaling in myogenic progenitors. Consistent with in vivo data, constitutive overexpression of Wnt-1 or Wnt-3a inhibited the proliferation of both C2C12 and primary myoblasts. Surprisingly, Wnt-1 and Wnt-3a overexpression up-regulated BMP-4, and inhibition of BMP-4 by shRNA or recombinant Noggin protein rescued the myogenic inhibitory effect of Wnt-1 and Wnt-3a. Importantly, Wnt-3a or BMP-4 recombinant proteins promoted slow myosin heavy chain expression during myogenic differentiation of fetal myoblasts.
\end{abstract}

Conclusions: These results demonstrate a novel interaction between canonical Wnt and BMP signaling that induces myogenic differentiation towards slow muscle phenotype.

Keywords: Skeletal muscle, Fetal myoblasts, Canonical Wnt signaling, BMP4 signaling, Differentiation, Slow muscle specification

\section{Background}

Skeletal muscles of the trunk and limb, except for some craniofacial and esophageal muscles, are derived from somites during embryonic development [1-3]. Specification of somitic cells into myogenic lineages is regulated by positive and negative signals from the surrounding tissues. Wnt signaling induced by Wnt-1, -3a, -4, -6, -7a and 11 from dorsal neural tube or ectoderm is critical for the induction, initiation and progression of myogenesis in the presomitic mesoderm and early somites (Reviewed in $[4,5])$. Within the embryonic myogenic progenitors, Wnt also regulate the expression of Pax3/7, MyoD and Myf5,

\footnotetext{
* Correspondence: mrudnicki@ohri.ca

${ }^{1}$ Sprott Center for Stem Cell Research, Ottawa Hospital Research Institute, 501 Smyth Road, Ottawa, ON K1H 8L6, Canada

Full list of author information is available at the end of the article
}

key transcription factors involved in myogenesis [6-10]. Importantly, genetic knockout studies have clearly demonstrated the requirement of several Wnt molecules and $\beta$-catenin in the normal development of skeletal muscles $[11,12]$. These diverse functions of Wnt are mediated by both a canonical signaling pathway that requires stabilization and nuclear translocation of $\beta$-catenin, and noncanonical pathway that is independent of $\beta$-catenin [5]. Therefore, canonical and non-canonical Wnt signaling pathways play multiple essential roles in embryonic myogenesis.

Wnt signaling is also involved in the regulation of postnatal satellite cell function and skeletal muscle regeneration. Satellite cells are muscle resident stem cells responsible for postnatal regeneration of injured muscles. 
During muscle regeneration, Wnt-5a and Wnt-7a induce muscle resident $C D 45^{+}$stem cells to undergo myogenic specification and differentiation [13]. Wnt-7a also acts through the non-canonical Wnt signaling pathway to stimulate the symmetric expansion of satellite cells and promote skeletal muscle hypertrophy $[14,15]$. Similarly, canonical Wnt activation was shown to induce satellite cell proliferation during skeletal muscle regeneration [16]. However, contradictory results showing that activation of canonical Wnt signaling is necessary to counteract Notch signaling to induce myogenic differentiation were also reported [17]. Furthermore, in the aged niche, elevated systemic Wnt molecules impede myogenic differentiation and facilitate satellite cell fate conversion to fibroblastic cell lineages [18].

Adult skeletal muscles contain heterogeneous types of muscle fibers that can be broadly divided into slow- and fast-twitch myofibers [19]. In the limb muscles, the slowtwitch myofibers express type I myosin heavy chain (MyHC), while the fast-twitch myofibers express type IIa, IIx and IIb MyHC isoforms [19]. During early stages of myofiber generation in chicks, Wnt-11 promotes fast myofiber formation, whereas Wnt-5 enhances slow myofiber generation [20]. Wnt-4 similarly stimulated fast myofiber formation in chicks [21]. Activation of $\beta$-catenin induced myofiber hypertrophy followed by degeneration of fast myofibers in zebrafish [22]. In mice, depletion of $\beta$-catenin during myogenesis in Pax7-lineage cells led to reduced slow myofibers and overall reduction of muscle mass [23]. Interestingly, expression of stabilized $\beta$-catenin in Pax7 lineage cells also led to reduced myogenesis but increased slow myofibers [23]. These studies suggest that Wnt signaling plays diverse roles in regulating slow- versus fast-twitch myofiber formation during development. However, embryonic lethality of $\beta$-catenin deficient mice or over-expressing mice precludes analysis of Wnt signaling in postnatal muscles. In addition, how canonical Wnt signaling regulates myofiber types has remained unclear.

Myofibers in postnatal skeletal muscles retain an adaptive capacity to switch between slow- and fast-twitch properties that largely depend on motoneuron activity [19]. Wnt signaling also regulates the establishment and maintenance of neuromuscular junctions that connect motor neurons and myofibers. In Drosophila and mice, Wnt secreted by presynaptic motoneurons interact with Agrin-MusK to induce assembly of postsynaptic endplates (Reviewed in [24]). There is evidence that neuromuscular junctions are phenotypically and functionally distinct in fast and slow muscles [25,26]. Whether Wnt signaling is differentially activated in slow and fast myofibers is completely unknown.

In this study, we used transgenic Wnt-reporter mice and mice with constitutively activated canonical Wnt signaling specifically within skeletal muscle to investigate the function of canonical Wnt signaling in muscle. We found that Wnt signaling is highly activated in prenatal muscle and rapidly declines in postnatal muscle, with some residual activation limited to the slow myofibers. Interestingly, Wnt signaling is highly activated proximal to motor endplates of slow myofibers. Wnt activation induced by stabilized $\beta$-catenin inhibits muscle differentiation and promotes slow muscle determination. At the molecular level, canonical Wnt signaling induces BMP-4 , which promotes expression of slow $\mathrm{MyHC}$. These novel results demonstrate that interplay between Wnt and BMP signaling regulates skeletal myogenesis and muscle fiber type.

\section{Methods \\ Plasmids}

Wnts cDNA subcloned from pLNCX-HA-Wnt constructs (kindly gifted from Dr. Jan Kitajewski) to pHAN-puro retrovirus vector by PCR $[27,28]$. Wnt-10b and TCF-4B cDNA were obtained from Thermo Fisher Scientific Open Biosystems (Waltham, MA, USA). Super TOPFlash was kindly gifted from Dr. Moon [29]. The mouse BMP-4 shRNA pLKO.1-puro (RMM3981-9595140) was obtained from Openbiosystems. Scramble shRNA pLKO.1-puro (Addgene Plasmid \#1864), MD2.G (Addgene Plasmid \#12259) and psPAX2 (Addgene Plasmid \#12260) were obtained from Addgene (Cambridge, MA, USA).

\section{C2C12 myoblasts, single myofibers and isolation of primary myoblasts}

C2C12 cells are purchased from American Type Culture Collection (ATCC) (Manassas, VA, USA) and cultured in DMEM with $10 \%$ FBS and antibiotics. Cells are induced to differentiation upon $80 \%$ confluence by serum withdrawal (DMEM with $2 \%$ horse serum). Primary myoblasts were isolated from the hind limb of two- to three-monthold wild type mice as previously described [27]. Single myofibers were isolated from flexor digitorum longus (EDL) muscle by collagenase digestion. The single fibers were immediately fixed in $4 \%$ paraformaldehyde (PFA), permeabilized by Triton-X100, stained with primary antibodies for $\beta$-gal (Life Technologies, Carlsbad, CA, USA), MyHC (MF-20, Developmental Studies Hybridoma Bank [DSHB], Iowa, Iowa, USA), slow MyHC (DSHB) and FITC-conjugated $\alpha$-bungarotoxin (BTX) (Sigma-Aldrich, St. Louis, MO, USA). Nuclei were counter stained with 4',6-diamidino-2-phenylindole (DAPI).

Fetal myoblasts were prepared from the legs of Myf5Cre/ROSA26-YFP embryos at E14.5. The legs are minced and digested by collagenase, dispase and DNaseI, as previously described for satellite cells isolation [30]. After collagenase procedure, collected cells were stained by alpha7-integrin antibody and anti-mouse IgG1Alexa648. Fetal myoblast were isolated by MoFlo (Dako, 
Glostrup, Denmark). Fetal myoblast were isolated by magnetic-antibody cell sorting (MACS) (Miltenyi Biotec, Bergish Gladbach, Germany). The isolated cells were incubated in culture dishes at $37^{\circ} \mathrm{C}$ for one hour to remove adherent cells, and the nonadherent cells were collected. The isolated fetal myoblasts cultured in DMEM/F10 medium with $20 \%$ FBS and bFGF.

\section{Retrovirus and lentivirus infection}

To prepare ecotropic retrovirus, Phoenix-eco packaging (kindly gifted from Dr. Gally Nolan) cells were transfected with retrovirus vectors using GeneJuice (Novagen EMD Chemicals, Madison, WI, USA). Viral supernatants were harvested 30 hours post transfection and used to infect $\mathrm{C} 2 \mathrm{C} 12$ cells in the presence of polybrene (Sigma, $8 \mathrm{mg} / \mathrm{ml}$ ) for 12 hours. Infected $\mathrm{C} 2 \mathrm{C} 12$ cells were then washed twice with phosphate-buffered saline (PBS), maintained in growth media and were selected 24 hours post-infection with puromycin (1.5 micro g/ml, Sigma). Lentivirus was packaged in 293T cells (ATCC CRL11268) transfected with the mouse BMP-4 or scramble shRNA pLKO.1-puro plasmids in addition to the pMD2. $\mathrm{G}$ and psPAX2 plasmids. Lentivirus was concentrated by ultracentrifuge and resolved in $\mathrm{PBS}(-)$ for virus infection. Infected $\mathrm{C} 2 \mathrm{C} 12$ and primary myoblast cells were then washed twice with PBS, and maintained in growth media.

\section{Gene expression analysis}

Total RNAs were prepared from $\mathrm{C} 2 \mathrm{C} 12$ and myoblast cells by TRIzol (Life Technologies). RNA samples were reverse transcribed using random hexamer and oligo dT mixed primers with SuperScriptII enzyme (Life Techonologies) according to the manufacturer's instructions. Reverse transcription reactions were diluted (1:10) with 10 $\mathrm{mM}$ Tris, $\mathrm{pH}$ 8.0, yielding master samples of reversetranscribed products. Real-time PCR reactions are previously described [27]. Real-time data were gathered using a system (MX4000; Agilent Technologies, Santa Clara, CA, USA) over 40 cycles $\left(30 \mathrm{~s}\right.$ at $90^{\circ} \mathrm{C}, 60 \mathrm{~s}$ at $58^{\circ} \mathrm{C}$ and $30 \mathrm{~s}$ at $72^{\circ} \mathrm{C}$ ) followed by a denaturation curve from $54^{\circ} \mathrm{C}$ to $94^{\circ} \mathrm{C}$ in 30-s increments of $0.5^{\circ} \mathrm{C}$ to ensure amplification specificity. Threshold cycle $(\mathrm{Ct})$ values were calculated with the MX4000 software (Agilent Technologies, Santa Clara, CA, USA) by using moving window aver-aging and an adaptive baseline. Fold changes, other calculations and chart plotting were performed in Microsoft Excel (Redmond, WA, USA). The sequence of PCR primers is listed in Additional file 1: Table S1.

\section{Animal care}

Myf5-Cre [31] heterozygous mice were bred with ROSA26-YFP [32]. TCF-lacZ [33] and Ctnn1 exon3 floxed [34] mice were previously described. All mice are main- tained inside a barrier facility, and experiments were performed in accordance with the University of Ottawa regulations for animal care and handling.

\section{Immunofluorescence staining}

$\mathrm{C} 2 \mathrm{C} 12$ cells were incubated at $37^{\circ} \mathrm{C}$ for two hours with $10 \mu \mathrm{M}$ BrdU, then washed with PBS(-) and fixed with $2 \%$ PFA/PBS. The fixed cells were stained with DAPI for 15 minutes at room temperature, washed with $\operatorname{PBS}(-)$, and refixed with 2\% PFA for 5 minutes at room temperature. The refixed cells were treated with $2 \mathrm{~N} \mathrm{HCl}$ for 20 minutes at room temperature (RT), neutralized by washing with $0.1 \mathrm{M}$ borate buffer $\mathrm{pH}$ 8.5. The cells were permeablized with $0.2 \%$ Triton X-100 PBS(-), blocked with broking buffer, incubated with anti-BrdU antibody for 2 to 12 hours. After staining with the primary antibody, cells were washed with $\operatorname{PBS}(-)$, stained with antimouse IgG1-Alexa488, washed with PBS(-) and mounted on slide glass with Dako mounting buffer. Myoblasts were fixed in $4 \%$ PFA/PBS (-) for 5 minutes, blocked with $10 \%$ goat serum/PBS (-) for 10 minutes, and stained with $\mathrm{MyHC}$ slow, MyHC fast, MyHC pan, antimouse IgG1-Alexa568, anti-mouseIgG2b-Alexa648 (Life Technologies) and DAPI (Sigma). Slides were mounted in SlowFade Light antifade Kit Component A (Molecular Probes) and analyzed with a Bio-Rad confocal laser scanning microscope (model MRC-1024) (Bio-Rad Laboratories, Hercules, CA, USA).

\section{ALP, X-gal and NADH-TR staining}

Cells were fixed 2\% PFA and washed with PBS(-), then stained by alkaline phosphatase (ALP) buffer (100 mM Tris- $\mathrm{HCl} \mathrm{pH}$ 9.5, $100 \mathrm{mM} \mathrm{NaCl}, 50 \mathrm{mM} \mathrm{MgCl}_{2}$ ) containing $4.5 \mu \mathrm{l}$ nitro-blue tetrazolium chloride (NBT) and $3.5 \mu \mathrm{l}$ 5-bromo-4-chloro-3-indolyl phosphate (BCIP) per $1 \mathrm{ml}$ of ALP buffer at $37^{\circ} \mathrm{C}$. Isolated whole embryos and tissue were fixed in $2 \%$ PFA for 3 hours at $4^{\circ} \mathrm{C}$ and permeablized with $\mathrm{X}$-gal staining buffer $(0.1 \mathrm{M}$ phoshate buffer ( $\mathrm{pH}$ 7.3), $2 \mathrm{mM} \mathrm{MgCl}_{2}, 0.01 \%$ sodium deoxycholate, $0.02 \%$ Nonidet P-40) for 2 hours at $37^{\circ} \mathrm{C}$ and stained by X-gal staining buffer with $0.1 \%$ X-gal, $5 \mathrm{mM}$ potassium ferricyanide and $5 \mathrm{mM}$ potassium ferrocyanide at $37^{\circ} \mathrm{C}$ for 2 to 24 hours.

The isolated muscle tissues were placed directly into optimal cutting temperature (OCT) compound and frozen in deep cold isopentane with ethanol and dry ice. The muscle tissues were cut $16 \mu \mathrm{m}$ by cryostat and dried in room temperature. The muscle sections were incubated with NADH-TR staining solution $(0.8 \mathrm{mg} / \mathrm{ml}$ $\mathrm{NADH}$ and $1 \mathrm{mg} / \mathrm{ml} \mathrm{NBT}$ in $50 \mathrm{mM}$ Tris-HCl (pH 7.6) at $37^{\circ} \mathrm{C}$. The stained muscle sections were washed with deionized water, unbound NBT was removed by acetone solution, then the sections were re-washed with deionized water and mounted with a coverslip. 


\section{Luciferase assays}

Myoblasts in 24-well plates were transfected with the plasmids indicated and $50 \mathrm{ng}$ pRL-PGK using Lipofectamine (Invitrogen). Transfected cells were harvested around 24 hours after transfection, and luciferase activities in the cell extracts were measured according to the manufacturer's instructions (Promega, Fitcburg, WI, USA) in a luminometer (Microplate luminometer LB96V (EG\&G Berthold Technologies, Bad Wildbad, Germany). Luciferase activities as indicated by arbitrary unit were normalized by sea urchin luciferase activities in each sample. All experiments were repeated at least three times, and the averages of more than three independent experiments with standard deviations are shown as bars [35].

\section{Western blots}

Infected $\mathrm{C} 2 \mathrm{C} 12$ cells were grown in $60-\mathrm{mm}$ dishes, washed twice with PBS and lysed in $100 \mathrm{~mL}$ radioimmunoprecipitation assay (RIPA) buffer $(50 \mathrm{mM}$ Tris $\mathrm{HCl}$, pH 7.5; $150 \mathrm{mM} \mathrm{NaCl} ; 0.5 \%$ Nonidet P-40; $0.1 \%$ deoxycholate) containing protease inhibitor cocktail (Roche Applied Science, Penzberg, Germany) [35]. Cell extracts were collected and spun in a microcentrifuge at 13,000 rpm for 5 minutes. Total proteins ( $5 \mathrm{mg}$ ) were separated on 10\% SDS-PAGE and transferred to ImmobilonP (EMD Millipore Corporation, Billerica, MA, USA). The membranes were probed with primary antibodies, followed by horseradish peroxidase (HRP)-conjugated secondary antibodies at 1:5,000 (Bio-Rad Laboratories), and developed using ECL ${ }^{\mathrm{TM}}$ Plus (GE Healthcare, Chalfont St. Giles, United Kingdam). Membranes were exposed to BIOMAX film (Eastman Kodak, Rochester, NY, USA). Primary antibodies used in this work: anti-MyoD (5.8A, BD Bioscience [San Jose, CA, USA]), anti-myogenin (F5D, DSHB), anti-MHC (MF-20, DSHB), anti-GAPDH (6C5, Life Technologies) and anti-.alpha;-tublin (Sigma-Aldrich).

\section{Results}

\section{Canonical Wnt signaling is activated during fetal} myogenesis and reduced in adult muscle

As the first step to investigate the function of Wnt signaling in myogenesis, we used the TCF-lacZ transgenic reporter mouse to examine the activity of the canonical Wnt signaling pathway in embryonic and adult muscles. The promoter of the LacZ transgene (encoding $\beta$ galactosidase, $\beta$-gal) contains multimerized TCF binding sites [33], a key downstream effector of canonical Wnt signaling. We analyzed $\beta$-gal activity, in embryonic and adult muscles by X-GAL staining (Figure 1 ). $\beta$-gal activity was detected in many muscles in E14.5 embryos (Figure 1A). Among those labeled, $\beta$-gal activity was particularly intense in both forelimb and hind limb muscles (Figure 1B), ventral body wall muscles (Figure 1C), dorsal spinotrapezius (Figure 1D) and intercostal muscles.
The number of muscles labeled with X-GAL was reduced in the forelimb and hand limb in P0 fetus (Figure 1E, F). In the adult, $\beta$-gal activity was undetectable in EDL and TA, muscles that are predominantly enriched with fast type myofibers, but was readily detected in part of the diaphragm (Figure 1G) and soleus (Figure 1H), muscles that are known to be enriched with slow myofibers. These data indicate that canonical Wnt signaling is strongly activated during fetal myogenesis and declined in postnatal muscles with some residual activity in slow myofibers.

Interestingly, the $\beta$-gal activity in postnatal slow muscle was especially strong at neuromuscular junctions (dark dotted staining patterns in the mid-belly of the muscles; Figure 1G-H). To further examine this phenomenon, we isolated single myofiber from the soleus muscles (containing about 50\% slow and 50\% fast myofibers) of Tcf-lacZ mice. The isolated single myofibers were fixed and stained with $\alpha$-bungarotoxin (BTX) and $\beta$-gal antibodies, together with slow-MyHC or pan-MyHC antibodies (Figure 2A-D). Consistently, strong $\beta$-gal immunoreactivity was detected proximal to motor endplates located within BTX stained neuromuscular junctions of type I fibers (Figure 2A, B), but not in type II myofibers (Figure 2C-D). Co-labeling whole mount muscle with slow and fast myosin heavy antibody confirmed the specific activation of $\beta$-gal in the in slow myofibers (Figure 2E, F). Overall, $97 \%$ of the $\beta-$ gal $^{+}$ myofibers co-expressed the slow $\mathrm{MyHC}$, where only $8 \%$ of the $\beta$-gal ${ }^{-}$myofibers co-expressed slow MyHC (Figure $2 G$ ). These data indicate that canonical Wnt signaling is highly activated at the neuromuscular junction area specifically in slow myofibers of adult skeletal muscles.

\section{Canonical Wnt signaling promotes formation of slow myofibers in vivo}

To confirm the role of canonical Wnt signaling in muscle fiber type specification in vivo, we took advantage of the Ctnnb ${ }^{\operatorname{lox}(\operatorname{ex} 3)}$ transgenic mice in which the exon 3 of $\beta$-catenin (Ctnnb) gene flanked by LoxP sites [34]. The exon 3 encodes serine and threonine residues that are normally phosphorylated GSK $3 \beta$, leading to the proteasomal degradation of $\beta$-catenin. Upon Cremediated excision of $C \operatorname{tnn} b$ exon $3, \beta$-catenin ${ }^{\Delta \mathrm{Ex} 3}$ is prevented from degradation (stabilized) and, therefore, constitutively active.

We first used Myf5-Cre to induce $\beta$-catenin ${ }^{\Delta \mathrm{Ex} 3}$ expression in myogenic progenitor cells. Myf5 is an early myogenic commitment marker during embryonic myogenesis [36]. Myf5-Cre/Ctnnb ${ }^{\operatorname{lox}(e x 3)}$ mice die at E15.5 with extremely reduced muscle mass (data not shown), thus precluding further analysis of myofiber types. We next used MCK-Cre to drive $\beta$-catenin ${ }^{\Delta \mathrm{Ex} 3}$ expression only in differentiated muscle cells. As expected, we detected 

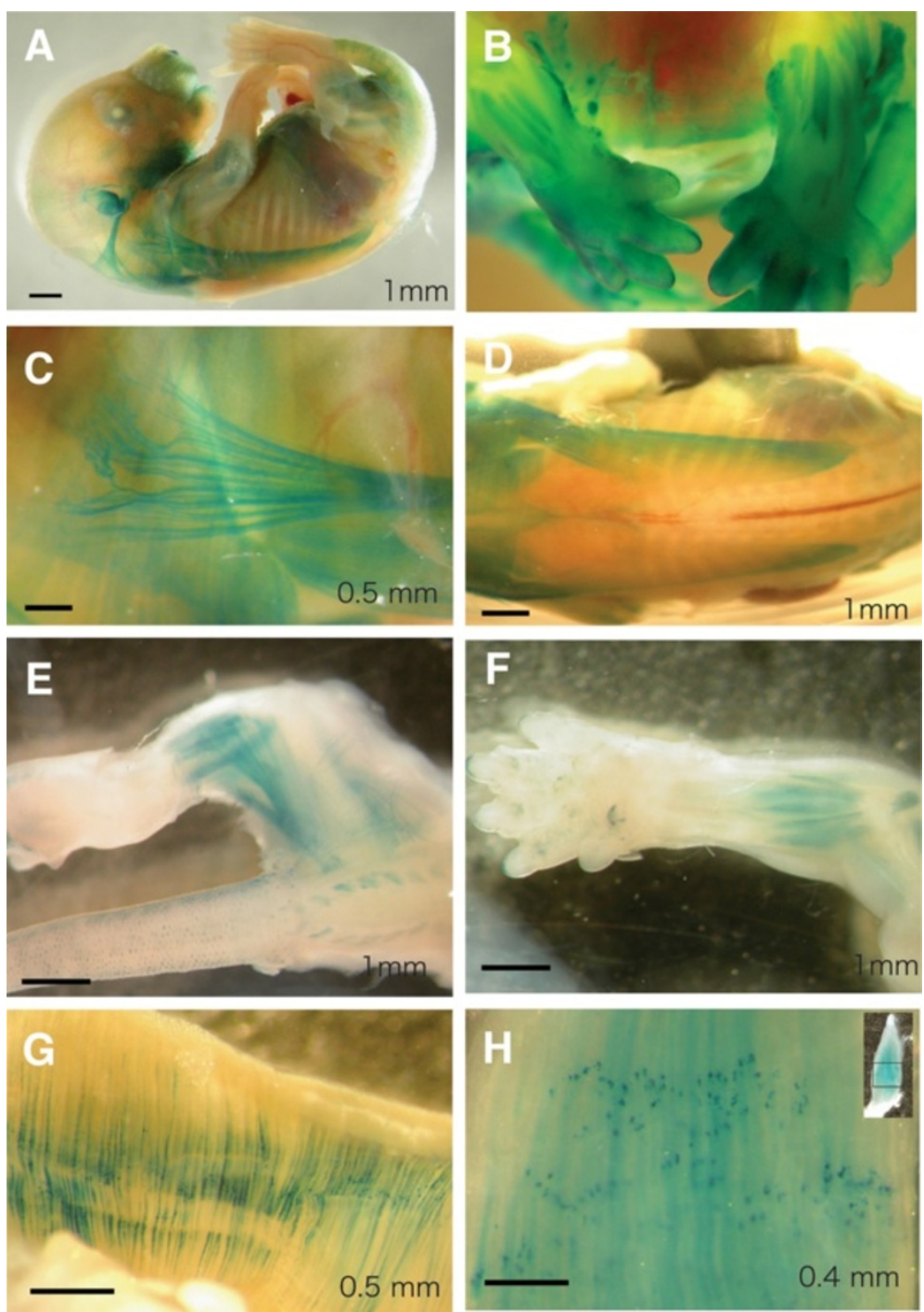

Figure 1 Activation of canonical Wnt signaling in embryonic, neonatal and adult skeletal muscles. Tcf-lacZ reporter mice were used to report activation of TCF promoter, the nuclear target of canonical Wnt signaling. X-gal staining (in blue) was used to reveal LacZ (b-gal) activity. (A) Whole mount staining of an E14.5 embryo. (B) Forelimbs and hind limbs at E14.5. (C) Ventral and (D) dorsal view of an E14.5 embryo revealing intensive staining in some muscles. (E) Hind limb and (F) forelimb at P0 (postnatal Day 0). (G) Diaphragm and (H) soleus muscles of adult mouse showing staining in a subset of myofibers with intensive signals at the neuromuscular junction area.

increased activation of canonical Wnt signaling in vivo in transgenic mice carrying MCK-Cre, Ctnnb ${ }^{\operatorname{lox}(\mathrm{ex} 3)}$ and TCFLacZ alleles. $\beta$-gal activity was detectable in TA muscles of the MCK-Cre/Ctnnb ${ }^{\text {lox(ex3) }} /$ TCF-LacZ mice, but not the $\mathrm{Ctnnb}^{\text {lox(ex3) }} /$ Tcf-lacZ littermate controls (Figure 3A, B).

In addition, we analyzed the oxidative activity of skeletal muscle myofibers using NADH-tetrazolium (NADH-TR) staining. Strikingly, the high and middle oxidative myofibers were increased in TA muscles of MCK-Cre/ $\mathrm{Ctnnb}^{\text {lox(ex3) }}$ mice $(\mathrm{n}=3,65.4 \pm 8.4 \%$ and $34.6 \pm 8.4 \%$, respectively) compared to the same muscle of wild type littermates ( $\mathrm{n}=3,52.7 \pm 5.3 \%$ and $19.0 \pm 3.8 \%$, respectively) (Figure 3C-E). By contrast, the number of the low oxidative myofibers was increased in wild type TA muscles $(\mathrm{n}=3,28.3 \pm 7.7 \%)$ compared relative to the mutant littermate (Figure 3C-E). As slow muscles are known to contain mainly oxidative myofibers and fast muscles are mainly low oxidative and glycolytic [19], these data provide in vivo evidence that canonical Wnt signaling promotes slow myofiber phenotype in the postnatal skeletal muscle.

\section{Canonical Wnt signaling inhibits proliferation of $\mathrm{C} 2 \mathrm{C} 12$ and primary myoblasts}

To identify the Wnt molecules that activate the canonical Wnt signaling pathway in muscle, we co-transfected 

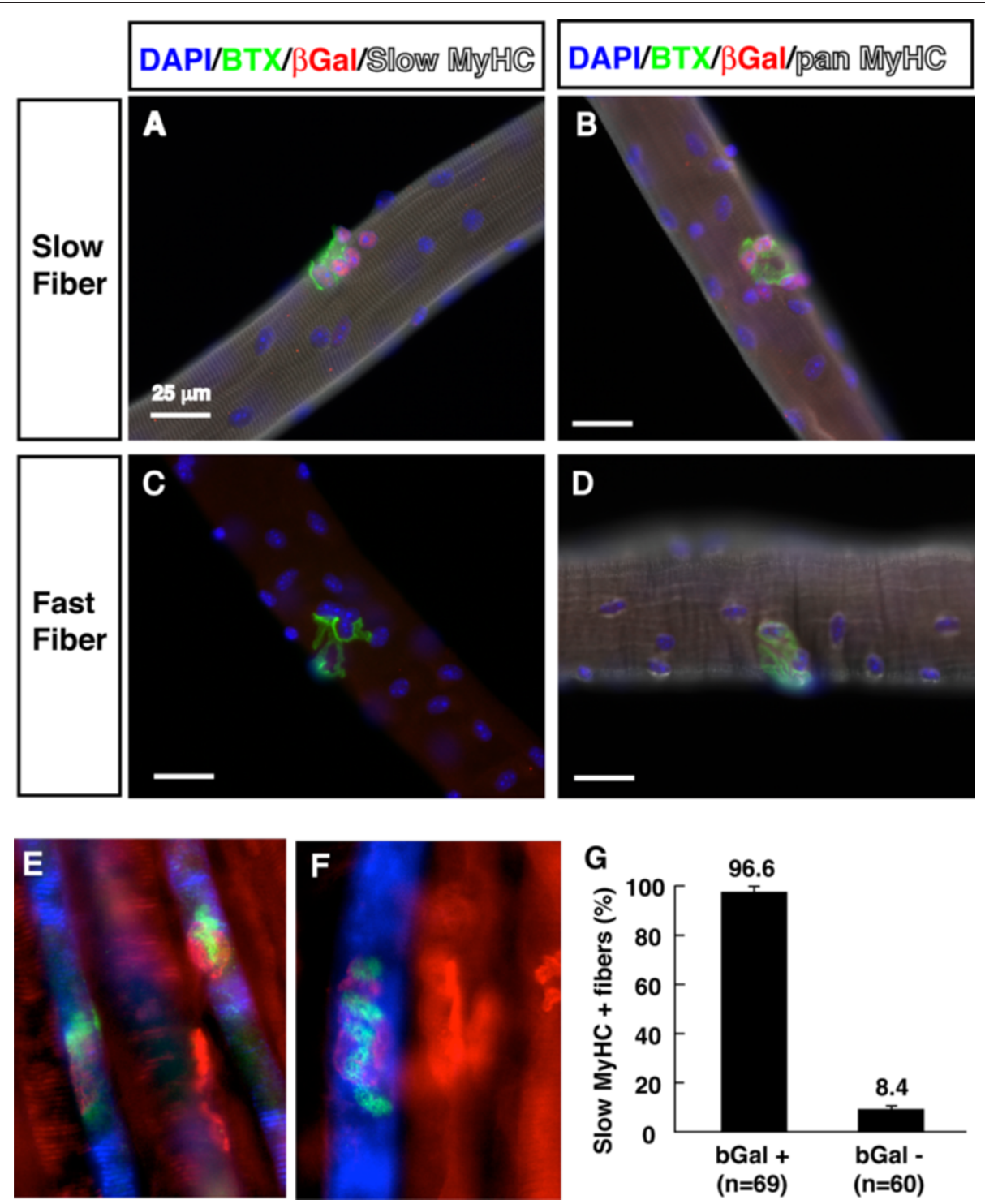

Figure 2 Activation of canonical Wnt signaling in adult slow myofibers. (A-D) Soleus myofibers from adult Tcf-lacZ mice were stained with antibodies for pan-myosin heavy chain (White) or slow myosin heavy chain (White), and $\beta$-gal (Red) and FITC conjugated BTX (Green). (E-F) Whole mount muscles co-stained with antibodies for slow (Blue) and fast (Red) myosin heavy together with beta-gal (Green) and BTX (Red). (G) Percentage of $\beta$-gal positive $(n=69)$ and negative $(n=60)$ myofibers that co-express slow myosin heavy chain.

candidate Wnt plasmids with the TCF/LEF reporter Super TopFlash. We found that Wnt-1 and Wnt-3a strongly activated ( $>400$ times increase in luciferase activity), Wnt-2 and Wnt-10b moderately activated ( $>20$ times increase in luciferase activity), and Wnt-2b and Wnt-4 weakly activated ( $>2$ times increase in luciferase activity) the canonical Wnt reporter (Additional file 2: Figure S1A). Other Wnts (Wnt-5a, Wnt-5b, Wnt-6, Wnt-7a, Wnt-7b, Wnt10a, Wnt-11) had no effect on the activation of the Super TopFlash reporter (Additional file 2, Figure S1A). We, therefore, used Wnt-1 and Wnt-3a to activate the canonical Wnt signaling in the following studies.
We next examined the function of both canonical and non-canonical Wnt signaling in cultured C2C12 myoblasts using retrovirus expressing various Wnts. Compared to mock controls, C2C12 cells overexpressing canonical Wnts (Wnt-1, -2, -2b, -3a, -10b) had a significantly decreased cell number after $96 \mathrm{~h}$ in culture (Additional file 2: Figure S1B). By contrast, C2C12 cells overexpressing non-canonical Wnts (Wnt-5a, -5b, -6, -7b, -11) had a moderately increased cell number compared to the control treated cells (Additional file 2: Figure S1B).

A reduced cell number in $\mathrm{C} 2 \mathrm{C} 12$ cells overexpressing Wnt-1, -2, -2b, $-3 \mathrm{a}$ and $-10 \mathrm{~b}$ indicates that canonical 

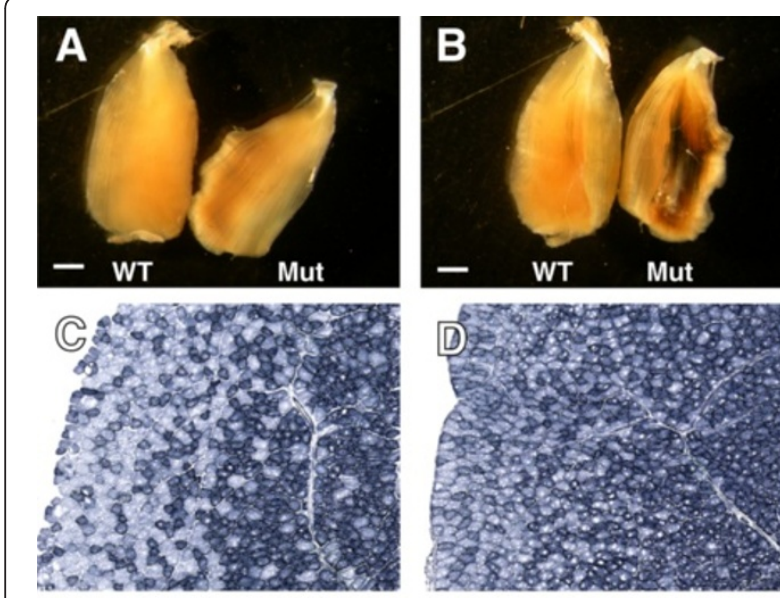

E

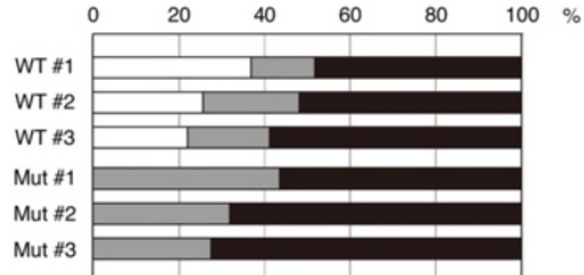

Figure 3 Muscle-specific constitutive activation of canonical Wnt signaling promotes oxidative myofiber phenotype. The MCK-Cre/Ctnnb lox(ex3) mice were used to express constitutively $\operatorname{active~} \beta$-catenin $^{\Delta \mathrm{ex} 3}$, which mimics canonical Wnt signaling. The MCK promoter-drive Cre expression limits Wnt activation only in mature skeletal muscles. (A-B) X-Gal staining (blue signal) of whole mount TA muscles of Ctnnb $b^{\text {lox(ex3) }} / \mathrm{Tcf}-\mathrm{LacZ}$ (WT) and MCK-Cre $/$ Ctnnb $b^{\text {lox(ex3) }} / T_{c f-L a c Z}$ (Mut) mice. Blue signal, indicative of canonical Wnt signaling, is only detectable in the Mut TA muscles (A and B represent the dorsal and ventral view of the same muscles). (C-D) NADH-TR staining of TA muscle sections from the WT (C) and Mut (D) mice. (E) Percentage of high oxidative (black), middle oxidative (gray) and low oxidative (white) myofibers of WT and Mut mice ( $n=3$, each). Scale bar: $1 \mathrm{~mm}$.

Wnt signaling inhibits cell proliferation. To confirm this, we analyzed the expression of Ki-67 (Additional file 2: Figure S1 C-E), a nuclear antigen specifically expressed in S, G2 and $M$ phase cells. The Wnt-1 and -3a expressing $\mathrm{C} 2 \mathrm{C} 12$ cells had decreased Ki-67 expression (Additional file 2: Figures S1 D-E, and 4I). In addition, we examined incorporation of BrdU, a thymidine analog that is incorporated into proliferating cells during the $\mathrm{S}$ phase. Proliferating $\mathrm{C} 2 \mathrm{C} 12$ cells were incubated with BrdU for one hour and then fixed for BrdU staining (Additional file 2: Figure S1F-H). Consistently, BrdU incorporation was reduced in the Wnt-1 and -3a expressing $\mathrm{C} 2 \mathrm{C} 12$ cells (Additional file 2: Figure S1G-H, J). Furthermore, we analyzed proliferation of skeletal muscle-derived primary myoblasts cultured with recombinant Wnt-3a protein $(50 \mathrm{ng} / \mathrm{ml})$ for 24 hours. Both Ki67 expression (Additional file 3: Figure S2A-C) and BrdU uptake (Additional file 3: Figure S2D-F) were decreased in the presence of Wnt-3a protein. These results indicate that canonical Wnt signaling suppressed the proliferation of $\mathrm{C} 2 \mathrm{C} 12$ cells and adult primary myoblasts.

To test if Wnt signaling has any effect on myogenic differentiation, we induced $\mathrm{C} 2 \mathrm{C} 12$ myoblasts to differentiate. Upon serum withdrawal, control myoblasts exited the cell cycle and fused to form myotubes with uniform morphology (Additional file 4: Figure S3A). By contrast, Wnt-1 and Wnt-3a expressing C2C12 cells formed very few myosin heavy chain (MyHC) positive myotubes (Additional file 4: Figure S3B-C). In addition, these myotubes were morphologically abnormal: they were short and chubby (Additional file 4: Figure S3B-C). As previously reported, non-canonical Wnt7a overexpression led to the formation of large myotubes resembling the muscle hypertrophy phenotype (Additional file 4: Figure S3D). We further examined by Western blotting the expression of two myogenic differentiation markers, myogenin and MyHC. Compared to the control, Wnt-1 and -3 a robustly inhibited the expression of Myogenin and $\mathrm{MyHC}$ at 48 hours, 72 hours and 96 hours postinduction of differentiation (Additional file 4: Figure S3E). Intriguingly, the non-canonical Wnt-7a not only increased Myogenin and MyHC protein levels, but also induced their earlier expression at 24 hours (Additional file 4: Figure S3E). However, recombinant Wnt-3a protein at $50 \mathrm{ng} / \mathrm{ml}$ had no effect on the differentiation of neither $\mathrm{C} 2 \mathrm{C} 12$ myoblasts (not shown) nor fetal primary myoblasts (see Figure $4 \mathrm{H}$ ). Thus, these data indicate that prolonged high-level constitutive activation of Wnt-1 and Wnt-3a in $\mathrm{C} 2 \mathrm{C} 12$ cells suppresses myogenic differentiation.

\section{Canonical Wnt signaling activates BMP signaling in $\mathrm{C} 2 \mathrm{C} 12$ myoblasts}

The reduced myogenic differentiation of Wnt-1 and Wnt-3a overexpressing $\mathrm{C} 2 \mathrm{C} 12$ myoblasts prompted us to analyze the alternative differentiation fate of these cells. Previous studies show that $\mathrm{C} 2 \mathrm{C} 12$ cells can also differentiate into osteogenic lineage [37], which express ALP. Surprisingly, Wnt-1, Wnt-3a and Wnt-10b overexpression strongly induced ALP immunochemical signals (Additional file 5: Figure S4A-F). By contrast, expression of non-canonical Wnt-5a and Wnt-7a did not affect ALP expression (Additional file 5: Figure S4D-E). Quantitative analysis confirms that Wnt-1 and Wnt-3a increased ALP enzyme activity by 15 - and 30 -fold, respectively (Additional file 5: Figure S4G).

To examine if Wnt-induced ALP activity was dependent on $\beta$-catenin, which mediates canonical Wnt signaling, we transduced $\mathrm{C} 2 \mathrm{C} 12$ cells with a dominant negative TCF-4b (DN-TCF-4b) that lacks the $\beta$-catenin binding site. As expected, DN-TCF-4b suppressed both 

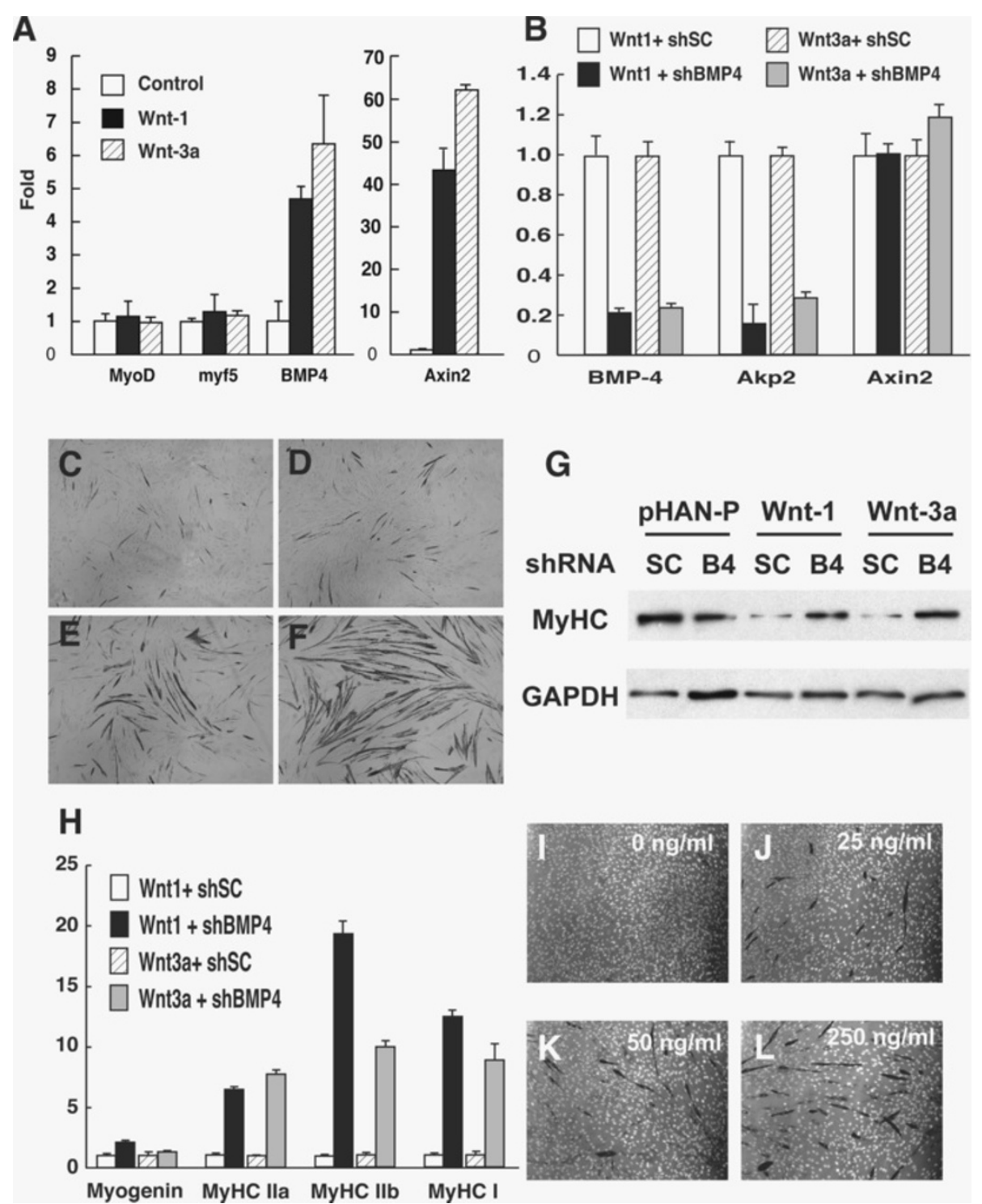

Figure 4 Canonical Wnt signaling induces BMP-4 in C2C12 myoblasts. (A) qPCR analysis of the relative expression of various genes in C2C12 myoblasts overexpressing Wnt-1 and Wnt-3a under growth condition. (B) BMP-4 knockdown reduced the expression of alkaline phosphatase (Akp2) but not the Axin2 gene. (C-F) BMP-4 shRNA rescued the myogenic differentiation of Wnt-1 and Wnt-3a infected C2C12 cells. (C-D) Wnt-1 and Wnt-3a overexpressing myoblasts treated with Scramble shRNA; (E-F) Wnt-1 and Wnt-3a overexpressing myoblasts treated with BMP-4 shRNA. Black signaling is myosin heavy chain antibody staining reacted with 3, 3'-diaminobenzidine (DAB) substrate. (G) Western blotting showing myosin heavy chain protein expression after BMP-4 shRNA treatment. (H) qPCR analysis of myosin heavy chain gene expression. (I-L) BMP-4 antagonist Noggin-Fc dose-dependently rescued the myogenic differentiation of Wnt-3a infected C2C12 cells. The myosin heavy chain was labeled in black and nuclei were labeled in white.

Wnt-1 and Wnt-3a induced ALP activity in C2C12 cells (Additional file 5: Figure S4H). These data indicate that canonical Wnt signaling induces ALP expression via a $\beta$-catenin/TCF-dependent pathway in $\mathrm{C} 2 \mathrm{C} 12$ cells.

That canonical Wnt signaling induces osteogenic ALP expression suggests a potential interaction between Wnt and BMP signaling pathways. We first examined BMP-4 gene expression using quantitative RT-PCR (qPCR) given its role in osteogenesis. Indeed, BMP-4 mRNA expression was increased by more than five-fold in Wnt1 and Wnt-3a overexpressing C2C12 cells (Figure 4A). In comparison, $M y f 5$ and $M y o D$ expression was not affected by Wnt-1 and Wnt-3a (Figure 4A). To ensure that canonical Wnt signaling is involved in the induction of BMP-4, we examined Axin2, a transcriptional target of $\beta$-catenin and canonical Wnt signaling [38]. Wnt-1 and Wnt-3a overexpression led to over 40X increase in the expression Axin2 (Figure 4A). 

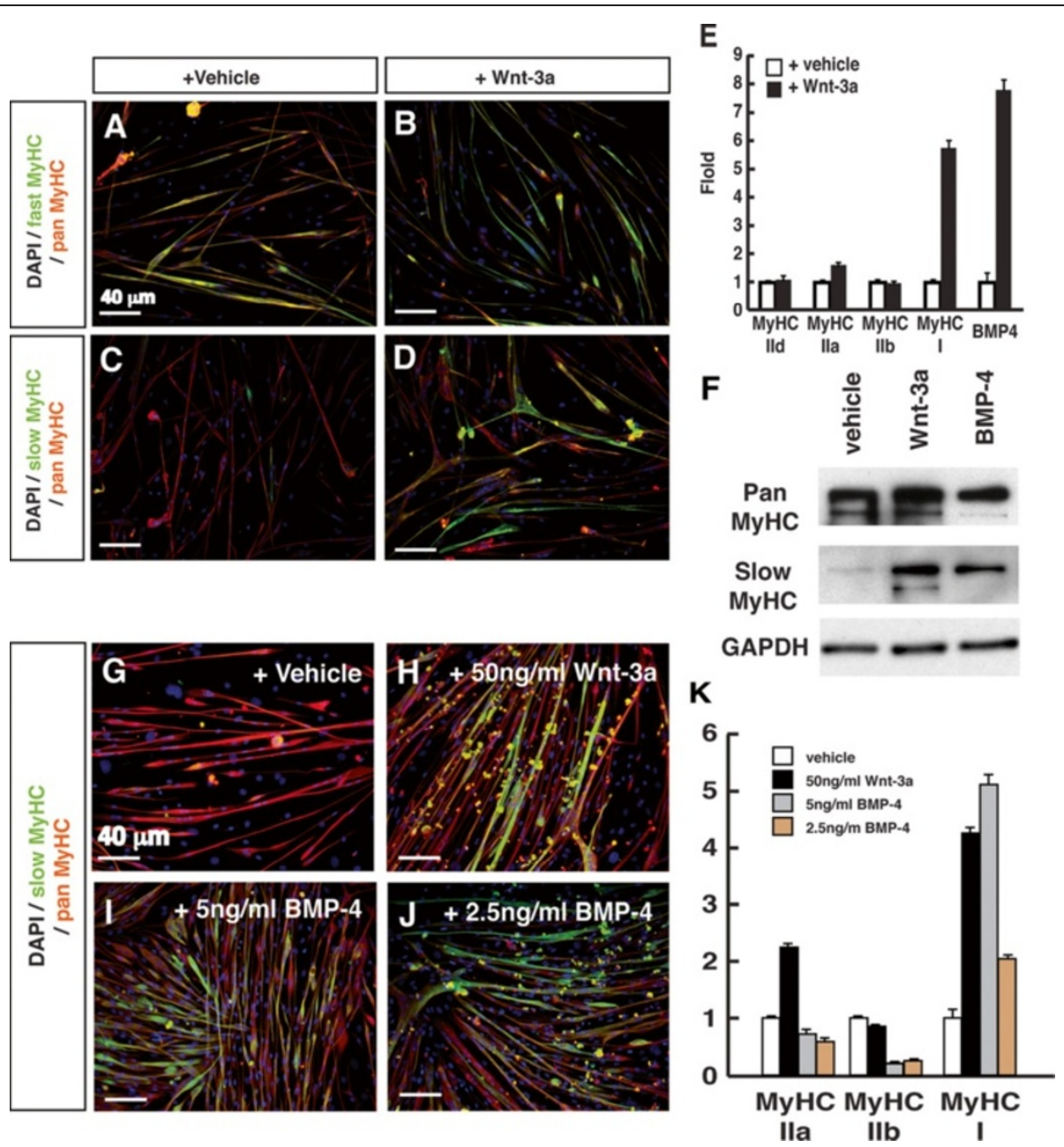

Figure 5 Wnt-3a induces slow myosin heavy chain expression in fetal myoblasts via BMP-4. Fetal myoblasts were isolated by fluorescenceactivated cell sorting (FACS) from E14.5-15.5 embryos of Myf5-Cre/Rosa26-YFP mice. FACS isolated fetal myoblasts were cultured and induced to differentiate for three days. (A-D) Immunofluorescence showing slow myosin heavy chain (green) expression in myotubes treated with vehicle control medium or Wnt-3a recombinant protein $(50 \mathrm{ng} / \mathrm{ml})$. (E) Relative expression of myosin heavy chain and BMP-4 genes based on qPCR analysis. (F) Western blotting showing slow myosin heavy chain protein expression. (G-J) Immunofluorescence showing slow- (green) and pan(red) myosin heavy chain expression in myotubes treated with vehicle control medium, Wnt-3a, and BMP-4 recombinant protein at concentration shown. (K) qPCR analysis showing relative expression of myosin heavy chain isoform genes. Scale bars: $40 \mu \mathrm{m}$.

We next asked if BMP-4 is necessary for proosteogenic effect of canonical Wnt signaling. We used lentiviral shRNA mediated knockdown of BMP-4 in $\mathrm{C} 2 \mathrm{C} 12$ cells. This approach resulted in nearly $80 \%$ reduction of BMP-4 transcripts (Figure $4 \mathrm{~B}$ ). Importantly, BMP4 knockdown reduced Wnt-1 and Wnt-3a induced $A k p 4$ (ALP gene) expression by more than 70\% (Figure 4B). By contrast, Axin2 mRNA levels were not decreased following knockdown of $B M P-4$ (Figure $4 \mathrm{~B}$ ), suggesting that $B M P-4$ signaling does not affect canonical Wnt signaling.

We further investigated if the anti-myogenic effect of canonical Wnt signaling is mediated by BMP-4 using the same shRNA knockdown approach. In the control groups (scrambled shRNA), there were only a few MyHC positive myotubes in the $\mathrm{C} 2 \mathrm{C} 12$ cells overexpressing Wnt-1 (Figure 4C) and Wnt-3a (Figure 4C). Knockdown of BMP4 remarkably increased the numbers of $\mathrm{MyHC}$ positive myotubes in Wnt-1 (Figure 4E) and Wnt-3a (Figure 4F) expressing $\mathrm{C} 2 \mathrm{C} 12$ cells. Consistent with this observation, $B M P-4$ shRNA rescued MyHC protein expression in Wnt1 and Wnt-3a overexpressing C2C12 cells (Figure 4G). Moreover, $B M P-4$ shRNA increased the mRNA levels of Myogenin, MyHC-IIa, MyHC-IIb and MyHC-I (Figure 4H). To confirm the above observations, we used recombinant Noggin protein, an antagonist of BMP, to block BMP activity. Noggin dose-dependently increased the number of MyHC positive myotubes in Wnt-3a expressing $\mathrm{C} 2 \mathrm{C} 12$ cells (Figure 4I-L). These data provide compelling evidence 
that canonical Wnt signaling inhibits myogenic differentiation through inducing BMP-4 signaling.

\section{Canonical Wnt signaling induces slow MyHC expression through BMP-4}

To directly examine the function of canonical Wnt signaling in muscle fiber type specification, we isolated embryonic myoblasts from E14.5 embryos by FACS. We employed positive selection for Myf5 and $\alpha 7$-integrin expression of myogenic cells from Myf5-Cre/ROSA-YFP embryos (Additional file 6: Figure S5). The purity of isolated fetal myoblasts was confirmed by immunostaining for Pax7 and desmin (Additional file 6: Figure S5). Embryonic myoblasts were cultured for one day before being induced to differentiate with or without the addition of Wnt-3a protein $(50 \mathrm{ng} / \mathrm{ml})$. In the absence of Wnt-3a, embryonic myoblasts differentiated into myotubes that expressed fast MyHC (Figure 5A), but not slow MyHC (Figure 5C) in agreement with previous studies [39]. By contrast, Wnt-3a treated fetal myoblasts differentiated into myotubes that expressed both fast (Figure $5 \mathrm{~B}$ ) and slow MyHC (Figure 5D).

To confirm this result, we analyzed MyHC-I mRNA expression by qPCR. The $M y H C-I$ mRNA level was upregulated 5.5-fold by Wnt-3a compared to control vehicle treatment (Figure 5E). Wnt3a also robustly induced the expression of slow MyHC-I at the protein level (Figure 5F). These results indicate that canonical Wnt signaling induces slow $\mathrm{MyHC}$ expression in fetal myoblasts.

As canonical Wnt signaling induced BMP-4, we further examined the role of BMP-4 in muscle fiber type specification. Consistent with our previous results in C2C12 cells (Figure 4A), Wnt-3a treatment of embryonic myoblasts induced a seven-fold increase in BMP-4 mRNA expression (Figure 5E). Next, we added recombinant BMP-4 to fetal myoblast cultures during differentiation. In the control treated with vehicle medium, newly formed $\mathrm{MyHC}^{+}$myotubes seldom expressed slow MyHC after three days of differentia tion (Figure 5G). In the presence of 2.5 to $5 \mathrm{ng} / \mathrm{ml}$ BMP-4, slow-MyHC ${ }^{+}$myotubes were abundantly visible (Figure 5I-J). The level of slow MyHC immunofluorescence induced by $5 \mathrm{ng} / \mathrm{ml} \mathrm{BMP}-4$ is similar to that induced by $50 \mathrm{ng} / \mathrm{ml}$ Wnt-3a (Figure $5 \mathrm{H}$ ), suggesting BMP-4 more potently induces slow MyHC expression. Western blotting showed that the slow MyHC protein expression level was indeed increased in the presence of BMP-4 (Figure 5F). In addition, qPCR analysis indicated that BMP-4 not only induced the slow $M y H C-I$ gene expression, but also robustly suppressed the fast $M y H C-I I b$ gene expression (Figure 5K). Collectively, these results indicate that canonical Wnt signaling acts through
BMP-4 to induce slow MyHC expression during embryonic myoblast differentiation.

\section{Discussion}

In this study, we use genetic, cell culture and molecular biology approaches to dissect the function of canonical Wnt signaling in myogenic differentiation and skeletal myofiber types. We show that the canonical Wnt signaling is most active during perinatal myogenesis and only activated in slow myofibers with high activity at the neuromuscular junction area in mature muscles. Constitutive activation of $\beta$-catenin, the canonical Wnt signaling effector, leads to impaired myogenesis and an increased proportion of oxidative myofibers in the postnatal muscles. Importantly, Wnt-1 and Wnt-3a mediated downstream signaling activates BMP-4, which inhibits the overall proliferation of myoblasts and promotes myogenic differentiation towards slow muscle phenotype. These results establish a novel interaction between Wnt and BMP signaling that regulates muscle fiber type specification and maintenance.

The TCF-LacZ reporter mouse has been widely used in reporting activation of canonical Wnt signaling in various tissues/cells $[11,40]$. Strong LacZ expression in specific muscles during embryonic and fetal myogenesis indicates activation of canonical Wnt signaling. Interesting, several muscles (spinotrapezius, body wall muscle and diaphragm) with high $\beta$-gal activity are known to be enriched with slow myofibers [19]. These results suggest a role of Wnt signaling in slow muscle generation and maintenance. Our in vivo results are consistent with previous studies in chick and fish embryos, in which canonical Wnt signaling was shown to promote slow muscle fate [20-22].

Our analysis of canonical Wnt signaling in adult mature muscles reveal several interesting points. First, $\beta$-gal activity is only detectable in muscles known to contain slow myofibers. This result confirms our observation in the developing embryonic muscles. Second, in contrast to embryonic muscle, where $\beta$-gal activity is evenly distributed within myofibers, the highest $\beta$-gal activity was within the slow myofibers was proximal to the motor endplate (Figure 1G-H). This observation suggests that whereas in embryonic muscle the Wnt molecules are released from surrounding tissues [5], Wnt signaling in adult slow myofibers is most likely initiated by Wnt molecules from motor neurons that innervate these myofibers. In support of the notion that the motor neuron supplies Wnt molecules, we found that $\beta$-gal immunoreactivity was no longer detectable in slow myofibers after three days of suspended culture in vitro in the absence of neural innervation (data not shown).

Previous studies demonstrate that Wnt molecules released by motor neurons play key roles in the 
development of neuromuscular junctions. Specifically, interaction of Wnt and LRP is necessary for clustering of postsynaptic acetylcholine receptors (AchR) [24]. Our new results demonstrate that Wnt signaling is further required for the maintenance of neuromuscular junction in slow myofibers. Future study is needed to examine the functional significance of Wnt signaling in slow versus fast muscle fibers and identify the Wnt molecules released by slow and fast motor neurons.

Using Cre-inducible transgenic mice that express stabilizes $\beta$-catenin, we investigated the role of canonical Wnt signaling in embryonic myogenesis and postnatal muscle maintenance. When Myf5-Cre is used as the driver mouse, which is expressed in embryonic myogenic progenitors, we detected abnormal muscle development and perinatal lethality. This observation is consistent to recent studies using Pax7-Cre or Myogenin-Cre to stabilize $\beta$-catenin, which also results in lethality at P0 $[23,41]$. In these studies, constitutive activation of $\beta$ catenin in the myogenic progenitors and committed myocytes resulted in a shift of fetal myofibers to slow muscle phenotype, and reduced myofiber size. However, the perinatal lethality of the Myf5-Cre, Myogenin-Cre and Pax7-Cre drive $\beta$-catenin activation precludes analysis of postnatal muscles. Using the Mck-Cre/ctnnb ${ }^{\operatorname{Lox}(e x 3)}$ mice, we found that adult muscles indeed have higher canonical Wnt activity based on the TCF-LacZ reporter assay. This verifies the utility of our mouse model.

Importantly, we found the adult fast (TA) muscles exhibited features of slow muscle phenotype as increased oxidative capacity. We also examined myosin heavy chain expression by immunohistochemistry but did not find any overt changes in myofiber types (data not shown). This result suggests that although Wnt signaling affects metabolic properties in the adult muscles, it is not sufficient to switch myosin heavy chain expression in the adult. This is expected since other factors, such as hormones and neural activity, can also influence myosin expression [19]. Together, our Mck-Cre/ctnnb ${ }^{\operatorname{Lox}(\text { ex3) }}$ model bypasses the premature lethality and provides novel insights of canonical Wnt signaling in regulating the oxidative capacity of adult muscles.

The observation that $\mathrm{C} 2 \mathrm{C} 12$ cells overexpressing Wnt-1 and Wnt-3a exhibited reduced proliferation and myogenic differentiation is quite intriguing. It could suggest that canonical Wnt/ $\beta$-catenin inhibits the proliferation and differentiation of myogenic cell lineages. This possibility would explain the reduced muscle mass phenotypes of the Myf5-Cre, Myogenin-Cre and Pax7-Cre induced stabilized $\beta$-catenin mice $[23,41]$. Alternatively, the result may also suggest that expression of Wnt-1 and Wnt-3a in the cell, independent of Frizzled receptor activation, is detrimental to cell growth and differentiation.
Interestingly, embryonic and fetal myoblasts seem to have different responses to canonical Wnt signaling [23], suggesting that the role of Wnt signaling, even in the same cell lineage, is also context dependent. Consistent with this notion, we show that the growth and differentiation of fetal primary myoblasts are not inhibited by recombinant Wnt-3a protein in culture. The osteogenic fate choice of Wnt-1 and Wnt-3a overexpressing C2C12 cells is in line with a recent report demonstrating fibroblastic lineage differentiation of satellite cells in response to high level of systemic Wnt molecules [18]. Thus, the observed effect of Wnt-1 and Wnt-3 in myogenic cell proliferation and differentiation is largely consistent with information in the literature.

We discovered a novel interaction between Wnt and BMP signaling in myoblasts. Bone morphogenetic proteins (BMPs) are multi-functional proteins belonging to the transforming growth factor beta (TGF $\beta$ ) superfamily. In zebrafish and frogs, BMP signaling inhibits the differentiation of muscle precursors in the dermomyotome and controls the number of myogenic cells [42,43]. During late myogenesis of mice, BMP signaling regulates the number of fetal myoblasts and satellite cells [44]. This action is through preventing the premature activation of MyoD while maintaining Pax3 expression. Therefore, BMPs may function to establish a sufficient number of myogenic progenitors before terminal differentiation.

Our cell culture results indicate that Wnt signaling induces BMP4, and BMP4 inhibition rescues the inhibitory effect of Wnt-1 and Wnt-3a on myogenesis. This result is in line with the above results in vivo. We further identify an unexpected role for BMP-4 in promoting slow muscle fate during fetal myogenesis. It is important to mention that low concentrations ( 1 to $5 \mathrm{ng} / \mathrm{ml}$ ) of BMP-4 protein were used in our study. Non-physiological, high concentrations of BMP will probably generate completely different effects [45]. Future studies should illustrate how BMP signaling regulates myosin gene expression.

Interestingly, in Drosophila larval neuromuscular junctions, retrograde BMP signaling controls synaptic growth [46]. The muscle-derived BMP modulates cytoskeletal dynamics and structural changes at presynaptic terminals. This forms a feedback system in which canonical Wnt molecules secreted from motor neurons not only induce formation of neuromuscular junctions, but also activate BMP-4 expression in the muscle. The muscle derived BMP-4 subsequently promotes development of presynaptic motor neuron terminals. Indeed, $\beta$-catenin stabilization in skeletal muscles (not limited to the neuromuscular junction area) results in increased motor axon number and excessive intramuscular nerve defasciculation and branching [41]. Taken together, our experiments have identified a novel interaction between canonical Wnt and BMP signaling that plays a role in myofiber type specification. 


\section{Conclusion}

Our study demonstrates that canonical Wnt-signaling controls the development of skeletal muscles via BMP-4 expression. High concentrations of BMP-4 have been previously established to inhibit myogensis and induce osteogenesis. We found that isolated fetal myoblasts do not normally form slow myofibers during myogenic differentiation in vitro. Strikingly, canonical Wnt-signaling induced low level BMP-4 expression that act to induce slow myofibergenesis. Therefore, we conclude that canonical Wnt and BMP signaling plays a hitherto unappreciated role in myofiber type specification during fetal myogenesis.

\section{Additional files}

Additional file 1: Table S1. The sequence of $P C R$ primers for $q P C R$ analysis is listed.

Additional file 2: Figure S1. Canonical Wnt signaling inhibits the growth and proliferation of C2C12 myoblast cells. (A) Relative luciferase activity of $\mathrm{C} 2 \mathrm{C} 12$ cells overexpressing various Wnt genes together with the SuperTop Flash reporter plasmid. (B) Graph of C2C12 cells transduced with retroviral Wnt plasmids. (C-E) The Ki-67 antibody staining and (F-H) BrdU incorporation of control, Wnt-1 and Wnt-3a transduced cells. (I) Percentage of Ki-67 positive cells in control, Wnt-1 and Wnt-3a transduced cells. (J) Percentage of BrdU incorporated cells in Control, Wnt-1 and Wnt-3a retrovirus infected C2C12 cells.

Additional file 3: Figure S2. Wnt-3a inhibits proliferation of adult primary myoblasts. (A-B) Ki-67 antibody staining of primary myoblasts treated with vehicle control $(\mathbf{A})$ and Wnt-3a recombinant protein (50 ng/ ml) (B). (C) Percentage of Ki67 positive cells. (D-E) BrdU incorporation of primary myoblast cells treated with control (D) and Wnt-3a protein (E). (F) Percentage of cells incorporated BrdU.

Additional file 4: Figure S3. Overexpression of Wnt-1 and Wnt-3a inhibits the myogenic differentiation of C2C12 myoblasts. (A-D) Immunofluorescence showing sarcomeric myosin heavy chain protein expression in $\mathrm{C} 2 \mathrm{C} 12$ cells transduced with retrovirus overexpressing $(\mathbf{A})$ Mock, (B) Wnt-1, (C) Wnt-3a and (D) Wnt-7a after four days of induced myogenic differentiation. (E) Western blotting analysis showing expression of myosin heavy chain (MyHC), myogenin and GAPDH in control, Wnt-1, Wnt-3a and Wnt-7a overexpression C2C12 myoblasts at different time points after induced to differentiate. GAPDH: Glyceraldehyde 3-phosphate dehydrogenase. Scale bar: $100 \mu \mathrm{m}$.

Additional file 5: Figure S4. Canonical Wnts (-1, $-3 a$ and 10b) induces alkaline phosphatase (ALP) activity in C2C12 cells. (A-F) ALP staining (Purple signal) of $\mathrm{C} 2 \mathrm{C} 12$ cells transduced retrovirus expressing empty vector (Mock) or Wnt vectors as indicated. (G) Relative ALP activity. (H) The dominant negative (DN) TCF-4 suppressed ALP activity in Wnt-1 and Wnt-3a expressing C2C12 cells.

Additional file 6: Figure S5. Isolation of fetal myoblasts from E14.5 embryos of Myf5-Cre/ROSA26-YFP mice by fluorescence activated cell sorting (FACS). (A-C) YFP expression in whole embryo (A), forelimb (B) and hindlimb (C) of E14.5-15.5 Myf5-Cre/ROSA26-YFP embryo. (D) Strategy for isolating fetal myoblasts by FACS. Whole limbs of Myf5-Cre /ROSA26-YFP embryos were minced and digested by collagenase and dispase. Single cells were stained with alpha7-integrin antibody and selected by YFP and alpha7-integrin expression. The sorted YFP and alpha7-integrin double positive cells were stained with antibody for Pax7 and desmin, makers of fetal myoblasts (nuclei were counterstained by DAPI in blue).

\section{Abbreviations}

BMP: Bone morphogenetic protein; TCF: T-cell factor; LEF: Lymphoid enhancer-binding factor; Pax: Paired box protein; FBS: Fetal bovine serum;
bFGF: Basic fibroblast growth factor; PFA: Paraformaldehyde; PGK: Phosphoglycerate kinase; GSK: Glycogen synthase kinase; MCK: Muscle creatine kinase; BrdU: Bromodeoxyuridine.

\section{Competing interests}

The authors declare no competing interests.

\section{Authors' contributions}

KK and MAR designed the research and wrote paper. SK performed the histology and tissue staining, and helped with paper writing. TMM provided the Ctnn1 exon3 floxed mice. All authors read and approved the final manuscript.

\section{Acknowledgments}

We thank Dr. Daniel Dufort for TCF-lacZ mice, Dr. Randall Moon and Dr. Valerie Wallace for DNA constructs. We thank members of the Rudnicki laboratory for their technical assistance and helpful discussions, and Dr. Makoto Sato for critical reading of the manuscript. KK was supported by a Postdoctoral Fellowship from Training Program in Regenerative Medicine in Canada.

\section{Author details}

'Sprott Center for Stem Cell Research, Ottawa Hospital Research Institute, 501 Smyth Road, Ottawa, ON K1H 8L6, Canada. ${ }^{2}$ Present Address: Department of Animal Sciences, Purdue University, 174B Smith Hall, 901 W State St, West Lafayette, IN 47907, USA. ${ }^{3}$ Department of Pharmacology, Kyoto University Graduate School of Medicine, Konoe, Yoshida, Sakyo, Kyoto 606-8501, Japan. ${ }^{4}$ Present address: Division of Cell Biology and Neuroscience, Department of Morphological and Physiological Sciences, Faculty of Medical Sciences, University of Fukui, 23-3, Matsuoka-Shimoaizuki, Eiheiji, Fukui 910-1193, Japan.

Received: 29 October 2012 Accepted: 15 February 2013 Published: 5 March 2013

\section{References}

1. Biressi S, Molinaro M, Cossu G: Cellular heterogeneity during vertebrate skeletal muscle development. Dev Biol 2007, 308:281-293.

2. Buckingham M, Vincent SD: Distinct and dynamic myogenic populations in the vertebrate embryo. Curr Opin Genet Dev 2009, 19:444-453.

3. Christ B, Ordahl CP: Early stages of chick somite development. Anat Embryol 1995, 191:381-396.

4. Cossu G, Borello U: Wnt signaling and the activation of myogenesis in mammals. EMBO J 1999, 18:6867-6872.

5. von Maltzahn J, Chang NC, Bentzinger CF, Rudnicki MA: Wnt signaling in myogenesis. Trends Cell Biol 2012, 22:602-609.

6. Abu-Elmagd M, Robson L, Sweetman D, Hadley J, Francis-West P, Munsterberg A: Wnt/Lef1 signaling acts via Pitx2 to regulate somite myogenesis. Dev Biol 2010, 337:211-219.

7. Tajbakhsh S, Borello U, Vivarelli E, Kelly R, Papkoff J, Duprez D, Buckingham M, Cossu G: Differential activation of Myf5 and MyoD by different Wnts in explants of mouse paraxial mesoderm and the later activation of myogenesis in the absence of Myf5. Development 1998, 125:4155-4162.

8. Borello U, Berarducci B, Murphy P, Bajard L, Buffa V, Piccolo S, Buckingham M, Cossu G: The Wnt/beta-catenin pathway regulates Gli-mediated Myf5 expression during somitogenesis. Development 2006, 133:3723-3732.

9. Ikeya M, Takada S: Wnt signaling from the dorsal neural tube is required for the formation of the medial dermomyotome. Development 1998, 125:4969-4976.

10. Otto A, Schmidt C, Patel K: Pax3 and Pax7 expression and regulation in the avian embryo. Anat Embryol (Berl) 2006, 211:293-310.

11. Aoki K, Taketo MM: Tissue-specific transgenic, conditional knockout and knock-in mice of genes in the canonical Wnt signaling pathway. Methods Mol Biol 2008, 468:307-331.

12. van Amerongen $R$, Berns $A$ : Knockout mouse models to study Wnt signal transduction. Trends Genet 2006, 22:678-689.

13. Seale P, Polesskaya A, Rudnicki MA: Adult stem cell specification by Wnt signaling in muscle regeneration. Cell Cycle 2003, 2:418-419.

14. Le Grand F, Jones AE, Seale V, Scime A, Rudnicki MA: Wnt7a activates the planar cell polarity pathway to drive the symmetric expansion of satellite stem cells. Cell Stem Cell 2009, 4:535-547. 
15. von Maltzahn J, Bentzinger CF, Rudnicki MA: Wnt7a-Fzd7 signalling directly activates the Akt/mTOR anabolic growth pathway in skeletal muscle. Nat Cell Biol 2012, 14:186-191.

16. Otto A, Schmidt C, Luke G, Allen S, Valasek P, Muntoni F, Lawrence-Watt D, Patel K: Canonical Wnt signalling induces satellite-cell proliferation during adult skeletal muscle regeneration. J Cell Sci 2008, 121:2939-2950

17. Brack AS, Conboy IM, Conboy MJ, Shen J, Rando TA: A temporal switch from Notch to Wnt signaling in muscle stem cells is necessary for normal adult myogenesis. Cell Stem Cell 2008, 2:50-59.

18. Brack AS, Conboy MJ, Roy S, Lee M, Kuo CJ, Keller C, Rando TA: Increased Wnt signaling during aging alters muscle stem cell fate and increases fibrosis. Science 2007, 317:807-810.

19. Schiaffino S, Reggiani C: Fiber types in mammalian skeletal muscles. Physiol Rev 2011, 91:1447-1531.

20. Anakwe K, Robson L, Hadley J, Buxton P, Church V, Allen S, Hartmann C, Harfe B, Nohno T, Brown AM, Evans DJ, Francis-West P: Wnt signalling regulates myogenic differentiation in the developing avian wing. Development 2003, 130:3503-3514.

21. Takata H, Terada K, Oka H, Sunada Y, Moriguchi T, Nohno T: Involvement of Wnt4 signaling during myogenic proliferation and differentiation of skeletal muscle. Dev Dyn 2007, 236:2800-2807.

22. Tee JM, van Rooijen C, Boonen R, Zivkovic D: Regulation of slow and fast muscle myofibrillogenesis by Wnt/beta-catenin and myostatin signaling. PLoS One 2009, 4:e5880.

23. Hutcheson DA, Zhao J, Merrell A, Haldar M, Kardon G: Embryonic and fetal limb myogenic cells are derived from developmentally distinct progenitors and have different requirements for beta-catenin. Genes Dev 2009, 23:997-1013.

24. Henriquez JP, Salinas PC: Dual roles for Wnt signalling during the formation of the vertebrate neuromuscular junction. Acta Physiol (Oxf) 2012, 204:128-136.

25. Chakkalakal JV, Nishimune H, Ruas JL, Spiegelman BM, Sanes JR: Retrograde influence of muscle fibers on their innervation revealed by a novel marker for slow motoneurons. Development 2010, 137:3489-3499.

26. Ogata T: Structure of motor endplates in the different fiber types of vertebrate skeletal-muscles. Arch Histol Cytol 1988, 51:385-424.

27. Ishibashi J, Perry RL, Asakura A, Rudnicki MA: MyoD induces myogenic differentiation through cooperation of its $\mathrm{NH} 2$ - and $\mathrm{COOH}$-terminal regions. J Cell Biol 2005, 171:471-482.

28. Soneoka Y, Cannon PM, Ramsdale EE, Griffiths JC, Romano G, Kingsman SM, Kingsman AJ: A transient three-plasmid expression system for the production of high titer retroviral vectors. Nucleic Acids Res 1995, 23:628-633.

29. Veeman MT, Axelrod JD, Moon RT: A second canon. Functions and mechanisms of beta-catenin-independent Wnt signaling. Dev Cell 2003, 5:367-377.

30. Kuang SH, Kuroda K, Le Grand F, Rudnicki MA: Asymmetric self-renewal and commitment of satellite stem cells in muscle. Cell 2007, 129:9991010.

31. Tallquist MD, Weismann KE, Hellstrom M, Soriano P: Early myotome specification regulates PDGFA expression and axial skeleton development. Development 2000, 127:5059-5070.

32. Srinivas $S$, Watanabe $T$, Lin CS, William CM, Tanabe $Y$, Jessell TM, Costantini F: Cre reporter strains produced by targeted insertion of EYFP and ECFP into the ROSA26 locus. BMC Dev Biol 2001, 1:4.

33. Mohamed OA, Clarke HJ, Dufort D: beta-catenin signaling marks the prospective site of primitive streak formation in the mouse embryo. Dev Dyn 2004, 231:416-424.

34. Harada N, Tamai Y, Ishikawa T, Sauer B, Takaku K, Oshima M, Taketo MM: Intestinal polyposis in mice with a dominant stable mutation of the beta-catenin gene. EMBO J 1999, 18:5931-5942.

35. Kuroda K, Han H, Tani S, Tanigaki K, Tun T, Furukawa T, Taniguchi Y, Kurooka $H$, Hamada $Y$, Toyokuni S, Honjo T: Regulation of marginal zone B cell development by MINT, a suppressor of Notch/RBP-J signaling pathway. Immunity 2003, 18:301-312.

36. Tajbakhsh S, Rocancourt D, Cossu G, Buckingham M: Redefining the genetic hierarchies controlling skeletal myogenesis: Pax-3 and Myf-5 act upstream of MyoD. Cell 1997, 89:127-138.

37. Nishimura R, Kato Y, Chen D, Harris SE, Mundy GR, Yoneda T: Smad5 and DPC4 are key molecules in mediating BMP-2-induced osteoblastic differentiation of the pluripotent mesenchymal precursor cell line C2C12. J Biol Chem 1998, 273:1872-1879.

38. Jho EH, Zhang T, Domon C, Joo CK, Freund JN, Costantini F: Wnt/betacatenin/Tcf signaling induces the transcription of Axin2, a negative regulator of the signaling pathway. Mol Cell Biol 2002, 22:1172-1183.

39. Hagiwara N, Yeh M, Liu A: Sox6 is required for normal fiber type differentiation of fetal skeletal muscle in mice. Dev Dyn 2007, 236:2062-2076.

40. Barolo S: Transgenic Wnt/TCF pathway reporters: all you need is Lef? Oncogene 2006, 25:7505-7511.

41. Liu Y, Sugiura Y, Wu FF, Mi WT, Taketo MM, Cannon S, Carroll T, Lin WC: beta-Catenin stabilization in skeletal muscles, but not in motor neurons, leads to aberrant motor innervation of the muscle during neuromuscular development in mice. Dev Biol 2012, 366:255-267.

42. Patterson SE, Bird NC, Devoto SH: BMP regulation of myogenesis in zebrafish. Dev Dyn 2010, 239:806-817.

43. Daughters RS, Chen Y, Slack JM: Origin of muscle satellite cells in the Xenopus embryo. Development 2011, 138:821-830.

44. Wang H, Noulet F, Edom-Vovard F, Tozer S, Le Grand F, Duprez D: Bmp signaling at the tips of skeletal muscles regulates the number of fetal muscle progenitors and satellite cells during development. Dev Cell 2010, 18:643-654.

45. Nakashima A, Katagiri T, Tamura M: Cross-talk between Wnt and bone morphogenetic protein 2 (BMP-2) signaling in differentiation pathway of C2C12 myoblasts. J Biol Chem 2005, 280:37660-37668.

46. Ball RW, Warren-Paquin M, Tsurudome K, Liao EH, Elazzouzi F, Cavanagh C, An BS, Wang TT, White JH, Haghighi AP: Retrograde BMP signaling controls synaptic growth at the NMJ by regulating trio expression in motor neurons. Neuron 2010, 66:536-549.

doi:10.1186/2044-5040-3-5

Cite this article as: Kuroda et al: Canonical Wnt signaling induces BMP-4 to specify slow myofibrogenesis of fetal myoblasts. Skeletal Muscle 2013 3:5.

\section{Submit your next manuscript to BioMed Central and take full advantage of:}

- Convenient online submission

- Thorough peer review

- No space constraints or color figure charges

- Immediate publication on acceptance

- Inclusion in PubMed, CAS, Scopus and Google Scholar

- Research which is freely available for redistribution 